COMMENT:

\title{
GENE THERAPY FOR AGE-RELATED MACULAR DEGENERATION
}

\author{
Robert E MacLaren
}

\begin{abstract}
Nuffield Laboratory of Ophthalmology, Department of Clinical Neurosciences, University of Oxford, Oxford University Eye Hospital \& Moorfields Eye Hospital NHS Foundation Trust
\end{abstract}

Age-related macular degeneration (AMD) is the most common cause of untreatable blindness in the world and is increasing. ${ }^{1}$ Current therapies aim to prevent growth of the abnormal retinal blood vessels that may leak and cause rapid visual loss in the later stages of the disease. This is achieved by regular injections into the eye of molecules that block the activity of vascular-endothelial growth factor (VEGF). ${ }^{2}$ These drugs are expensive and the requirement for regular intraocular injections indefinitely puts a significant strain on healthcare resources. In this issue, Rakoczy and colleagues present 1 year results from a Phase I clinical trial to assess a single treatment, using gene therapy, as an alternative to regular injections for achieving long-term VEGF blockade. ${ }^{3}$

An adeno-associated viral (AAV) vector was used to deliver the gene encoding soluble Fms-related tyrosine kinase-1 (sFLT-1), a naturally occurring inhibitor of VEGF. A dose of $10^{10}$ increasing to $10^{11}$ genome particles of AAV.sFlt-1 was administered by a single injection under the peripheral retina in two groups of three patients (avoiding the central macula). The hypothesis is that transduced cells will produce sufficient sFlt-1 protein to diffuse into the vitreous and inactivate VEGF. ${ }^{4}$ Treatment effects were assessed by a reduction in the need for further injections of the standard treatment (ranibizumab) and improvements in retinal anatomy during the one year study period. Whilst there was no matched control group, comparisons to historical data from other studies was in keeping with a treatment effect from the procedure. There were no safety concerns, such as retinal ischaemia or atrophy of the retinal tissue, which has been a concern with prolonged antiVEGF therapy. ${ }^{5}$

This trial is notable in exploring the use of gene therapy as a means of providing a 'protein pump', which is particularly suited to the enclosed compartment of the eye. Whilst AAV gene therapy is established for gene replacement in single gene disorders, ${ }^{6}$ the use of gene therapy to reprogram genetically normal cells of the retina to take on additional functions opens a new chapter in potential AAV applications. One of the very first retinal gene therapy trials also used a viral vector as a pharmacological tool for the sustained delivery of a therapeutic protein to block VEGF in AMD. ${ }^{7}$ The key difference between that and the current trial is that the former study used an adenoviral (Ad) vector to deliver the VEGF antagonist, which was predicted to have only a temporary effect. ${ }^{7}$ Adeno-associated viral (AAV) vectors, in contrast, have evolved to evade the host immune system and have shown evidence of indefinite gene expression following human subretinal administration. ${ }^{8}$

Other anti-VEGF approaches in clinical trials include administering the AAV vector directly into the vitreous cavity rather than into the subretinal space (NCT01024998). Intravitreal injections are easier to perform, but most likely a higher vector dose would be needed and there could be more off-target effects due to transducing retinal ganglion cells and a greater risk of inflammation. ${ }^{9}$ The current study validates the subretinal approach. There are however outstanding questions, such as which promoter to use. Although not stated in this paper, the preclinical study of AAV.sFlt-1 used the cytomegalovirus (CMV) promoter, ${ }^{10}$ which can be subject to longterm silencing in the brain. ${ }^{11}$ Similarly it remains to be seen if a surgically-implanted slow release device, which is also currently in clinical trial, ${ }^{12}$ may yield more predictable levels of VEGF inhibition compared with gene therapy. In this regard, it would be helpful to know the variability in intravitreal levels of sFlt-1 when delivered by gene therapy. Where gene therapy has an unrivalled advantage over slow release devices, however, is when proteins need to be expressed within the cytoplasm of the cells in order to have a therapeutic effect. In AMD the ideal goal would be to prevent the disease altogether using AAV gene therapy, possibly by correcting genes encoding complement proteins with a single subretinal administration. That would be as close to a cure for AMD as we could get. 
Figure 1 - Life cycle of recombinant AAV.sFIt-1

(A) The plasmid DNA sequence used to clone the recombinant adeno-associated viral ( $R A A V)$ vector includes the original wildtype virus inverted terminal repeat (ITR) sequences at 5' and 3' ends to facilitate packaging of the viral DNA into the protein capsid. The sFlt-1 transgene (cDNA only without introns) is flanked by Promoter and a PolyA signals for the beginning and end of gene transcription. (B) The rAAV is manufactured in the laboratory in human embryonic kidney (HEK) cells. Single stranded DNA from the above sequence is packaged into the AAV capsid particles which are assembled around the ITR sequences to form the vector - an enclosed icosahedron of capsid protein. (C) After successful transduction of human cells, the vector capsid is removed and the transgene is converted back into double stranded DNA in the host nucleus. This DNA is stabilised by the ITR sequences which facilitate transcription of $m R N A$. The promoter and PolyA regions leave a short untranslated region (UTR) at either side of the sFlt1 coding sequence. The gene expression from the stabilised rAAV genome is long-lasting.

\section{References}

1. Bourne RR, Stevens GA, White RA, et al. Causes of vision loss worldwide, 1990-2010: a systematic analysis. Lancet Glob Health 2013; 1:e339-49.

2. Schmidt-Erfurth $U$, Chong V, Loewenstein A, et al. Guidelines for the management of neovascular agerelated macular degeneration by the European Society of Retina Specialists (EURETINA). Br J Ophthalmol 2014; 98:1144-67.

3. Rakoczy EP, Lai C-M, Magno AL, et al. rAAV Gene Therapy for Neovascular Age-Related Macular Degeneration: One year follow up of a Phase 1 Clinical Trial. Lancet 2015 IN PRESS

4. Bainbridge JW, Mistry A, De Alwis M, et al. Inhibition of retinal neovascularisation by gene transfer of soluble VEGF receptor sFlt-1. Gene Ther 2002; 9:320-6.

5. Grunwald JE, Daniel E, Huang J, et al. Risk of geographic atrophy in the comparison of age-related macular degeneration treatments trials. Ophthalmology 2014; 121:150-61.

6. Day TP, Byrne LC, Schaffer DV, Flannery JG. Advances in AAV vector development for gene therapy in the retina. Adv Exp Med Biol 2014; 801:687-93.

7. Campochiaro PA, Nguyen QD, Shah SM, et al. Adenoviral vector-delivered pigment epithelium-derived factor for neovascular age-related macular degeneration: results of a phase I clinical trial. Hum Gene Ther 2006; 17:167-76.

8. Testa F, Maguire AM, Rossi S, et al. Three-year follow-up after unilateral subretinal delivery of adenoassociated virus in patients with Leber congenital Amaurosis type 2. Ophthalmology 2013; 120:1283-91.

9. Kotterman MA, Yin L, Strazzeri JM, et al. Antibody neutralization poses a barrier to intravitreal adenoassociated viral vector gene delivery to non-human primates. Gene Ther 2015; 22:116-26.

10. Lai CM, Estcourt MJ, Himbeck RP, et al. Preclinical safety evaluation of subretinal AAV2.sFlt-1 in nonhuman primates. Gene Ther 2012; 19:999-1009.

11. Paterna JC, Moccetti T, Mura A, Feldon J, Büeler H. Influence of promoter and WHV post-transcriptional regulatory element on AAV-mediated transgene expression in the rat brain. Gene Ther 2000; 7:1304-11.

12. Humayun M, Santos A, Altamirano JC, et al. Implantable MicroPump for Drug Delivery in Patients with Diabetic Macular Edema. Trans/ Vis Sci Technol 2014; 3:5.

\section{Declaration}

The author is a Founder and Director of Nightstarx Ltd (Gibbs Building, 215 Euston Road, London NW1 2BE), a choroideremia gene therapy company established by the University of Oxford and funded by the Wellcome Trust through Syncona Partners. 